Semin Fetal Neonatal Med. 2013 February ; 18(1): 1-2. doi:10.1016/j.siny.2012.11.002.

\title{
Perinatal pharmacology
}

John van den Anker ${ }^{*}$ and

Division of Pediatric Clinical Pharmacology, Children's National Medical Center, Washington, DC, USA; Department of Pediatrics, George Washington University, School of Medicine and Health Sciences, Washington, DC, USA; Department of Pharmacology \& Physiology, George Washington University, School of Medicine and Health Sciences, Washington, DC, USA; Department Integrative Systems Biology, George Washington University, School of Medicine and Health Sciences, Washington, DC, USA; Intensive Care, Erasmus MC - Sophia Children's Hospital, Rotterdam, The Netherlands

\section{Karel Allegaert}

Neonatal Intensive Care Unit, University Hospitals Leuven, Belgium

In the eight years following the last issue of Seminars in Fetal and Neonatal Medicine devoted to perinatal pharmacology, significant progress has been made. To illustrate this, a PubMed search in early June 2012 for 'perinatal pharmacology' resulted in 6236 hits in the last 15 years, with a modest, progressive increase in consecutive five-year (1793 to 2130 to 2313 hits) periods. To put the registered clinical research activity in neonates into some perspective, the www.clinicaltrials.gov website contains 143580 registered studies throughout the world, of which only 2667 (1.8\%) have 'newborn' as a subpopulation (search performed June 2012).

In the current issue, the topics covered relate either to fetal (e.g. effects of drugs on the fetus), maternal-neonatal (e.g. breastfeeding) and neonatal [e.g. human immunodeficiency virus (HIV) therapy, antibiotics, analgesia] clinical pharmacology or they illustrate new emerging concepts (e.g. biomarkers). There is an intentional overlap with the previous issue to further illustrate the substantial progress made in these specific areas of perinatal pharmacology, and to provide suggestions for new research endeavors. The gain in knowledge during this last decade is associated with the appearance of new problems and challenges (e.g. maternal codeine and neonatal sedation, neonatal serotonin reuptake inhibitor syndromes, growing up as HIV patient, integration of pharmacogenetics with developmental pharmacology). ${ }^{1-4}$

However, we have to be aware that the incentives that resulted in the increased research activity and gain in knowledge are not acquired forever. ${ }^{5}$ Neither does the increased research activity automatically result in improved outcome, safer treatments or more effective dosing regimens for mothers and their newborns. ${ }^{6-8}$

\footnotetext{
"Corresponding author. address: jvandena@cnmc.org (J. van den Anker).

Conflict of interest statement: None declared.
} 
Following US initiatives (e.g. network of Pediatric Pharmacology Research Units, Obstetric Pharmacology Research Units), the European Union (EU) and the World Health Organization (WHO) have also been active in providing incentives to improve perinatal health through improving access to better, tailored medicines, including issues such as childfriendly, age-appropriate formulations (European Pediatric Formulation initiative, child-size drug campaigns, list of essential medicines). However, the legal supportive environment is still less developed in other parts of the world and is not fully secured in the regions where there are legal initiatives. ${ }^{5}$ Moreover, new research data do not automatically translate into changes in clinical care and benefits to the infants in the region where the clinical trials were conducted. The difficulties in the translation of research data to clinical care in part relate to financial burdens, ${ }^{5}$ but also to difficulties in extrapolating study data to the clinical setting and to limitations during implementation. ${ }^{6-8}$ Outside the USA, the balance between the financial potential benefits and perceived risks for the manufacturers often results in nonmarketing of the formulation. Retaining and fostering interest in pediatric medicines for all stakeholders involved (e.g. industry, academia, regulatory agencies, parents and patients, the society) is challenging, but remains crucial for success.

Moreover, extrapolation and implementation of study data to the clinical setting need further consideration. ${ }^{6-9}$ Enrollment of neonates in a clinical research study may not be representative for the population of patients for whom we care. Recently, it has again been shown that there are indeed significant outcome differences between enrolled and eligible but not enrolled patients, due to enrollment bias related to antenatal consent. ${ }^{6-8}$ Similarly, the concept of parental consent also introduces an additional bias. Respect for the rights of the individual patient is inherent to any medical research, but the proxy consent in neonatal research also introduces an additional bias as has recently been reported for the SUPPORT trial. Infants who were not enrolled in this study were less likely to be white, insured, exposed to prenatal care or to have attained a higher level of education compared with mothers of infants enrolled in this study. Such biases potentially result in limitations during extrapolation of the reported data to the unit-specific setting. ${ }^{6-8}$

Finally, perinatal medicine has had both great successes and failures if we evaluate the dissemination, integration and implementation of new treatment modalities or techniques. ${ }^{9}$ There is still important variability in the use of proven successful treatments such as prenatal lung maturation between different units as well as the continued use of treatments with documented serious adverse events such as the use of postnatal dexamethasone. As a consequence, future research in the field of perinatal pharmacology should also focus on issues related to the availability of drugs and medical devices. More specifically, there ought to be a delicate balance between on the one hand the real costs of development and production of drugs and devices used in perinatal medicine, and on the other hand a 'reasonable' compensation for the companies involved in this field. At this moment in time we see regularly outrageous requests for compensation of efforts in this arena that jeopardize the use of safe and effective drugs and devices in this vulnerable patient population. Finally, we need more clinical research in the area of translation of new discoveries into clinical practice, and in the area of translating new developments in academic clinical practice to the community pediatricians. 
In the meantime, we are grateful for the efforts made by all authors and research groups who contributed to this issue, and hope that the articles herein illustrate well the scope and importance of this interdisciplinary clinical research field. In addition we hope that many will reconsider their daily clinical routine activities, and that clinicians and clinicianscientists will be stimulated to collaborate as teams to assure that the outcomes of pregnant women and their offspring will further improve. ${ }^{10}$

\section{Acknowledgments}

Funding sources: Karel Allegaert is supported by the Fund for Scientific Research, Flanders (Belgium) (F.W.O. Vlaanderen) by a Fundamental Clinical Investigatorship (1800209N). Johannes van den Anker is supported in part by NIH grants (R01HD060543, K24DA027992, R01HD048689, U54HD071601) and FP7 grants TINN (223614), TINN2 (260908), and NEUROSIS (223060).

\section{References}

1. Leeder JS, Kearns GL, Spielberg SP, van den Anker J. Understanding the relative roles of pharmacogenetics and ontogeny in pediatric drug development and regulatory science. J Clin Pharmacol. 2010; 50:1377-87. [PubMed: 20150527]

2. Du, W.; Tutag Lehr, V.; Lieh-Lai, M., et al. An algorithm to detect adverse drug reactions in the neonatal intensive care unit: a new approach. J Clin Pharmacol. 2012 Jan 18. http://dx.doi.org/ 0091270011433327

3. Kearns GL. Beyond biomarkers: an opportunity to address the 'pharmacodynamic gap' in pediatric dug development. Biomark Med. 2010; 4:783-6. [PubMed: 21133695]

4. Koren G, Clark S, Matsui D. Drugs during pregnancy and lactation: new solutions to serious challenges. Obstet Gynecol Int. 2012; 2012:206179. [PubMed: 22529853]

5. Hoppu K, Anabwani G, Garcia-Bournissen H, et al. The status of paediatric medicines initiatives around the world - what has happened and what has not? Eur J Clin Pharmacol. 2012; 68:1-10. [PubMed: 21732178]

6. Whitney SN. The Python's embrace: clinical research regulation by institutional review boards. Pediatrics. 2012; 129:576. [PubMed: 22371469]

7. Rich W, Finer NN, Gantz MG, et al. Enrollment of extremely low birth weight infants in a clinical research study may not be representative. Pediatrics. 2012; 129:480-4. [PubMed: 22371462]

8. Ballard HO, Shook LA, Iocono J, Bernard P, Hayes D Jr. Parents' understanding and recall of informed consent information for neonatal research. IRB. 2011; 33:12-9. [PubMed: 21736138]

9. Soll RF. Evaluating the medical evidence for quality improvement. Clin Perinatol. 2010; 37:11-28. [PubMed: 20363445]

10. Allegaert, K.; Langhendries, JP.; van den Anker, JN. Educational paper: do we need neonatal clinical pharmacologists?. Eur J Pediatr. 2012. http://dx.doi.org/10.1007/s00431-012-1734-4 